\title{
Advances in the synthesis, characterisation, and mechanistic understanding of active sites in Fe-zeolites for redox catalysts.
}

Received 00th January 20xx, Accepted 00th January 20xx

DOI: $10.1039 / x 0 \times x 00000 x$

\author{
Max L. Bols, ${ }^{* a}$ Hannah M. Rhoda, ${ }^{b}$ Benjamin E. R. Snyder, ${ }^{b}$ Edward I. Solomon, ${ }^{* b}$ Kristine Pierloot, ${ }^{* c}$ \\ Robert A. Schoonheydt*a and Bert F. Sels*a
}

\begin{abstract}
The recent research developments on the active sites in Fe-zeolites for redox catalysis are discussed. Building on the characterisation of the $\alpha-\mathrm{Fe} / \alpha-\mathrm{O}$ active sites in the beta and chabazite zeolites, we demonstrate a bottom-up approach to successfully understand and develop Fe-zeolite catalysts. We use the room temperature benzene to phenol reaction as a relevant example. We then suggest how the spectroscopic identification of other monomeric and dimeric iron sites could be tackled. The challenges in the characterisation of active sites and intermediates in NOx selective catalytic reduction catalysts and further development of catalysts for mild partial methane oxidation are briefly discussed.
\end{abstract}

\section{Introduction}

Heterogeneous zeolite catalysts combine a thermally stable, chemically inert, solid and crystalline aluminosilicate framework with a high pore volume and surface area. This enables a high density of active sites on their surface for acid and/or redox catalysis. The crystalline zeolite offers several advantages over amorphous aluminosilicates: (1) the crystalline structure can be accurately determined by XRD and related techniques; (2) the well-defined pore structure can be used to influence catalysis through molecular sieving, shape selectivity, and transition state stabilization; (3) the Brönsted acid sites (BAS) of the zeolites are well-defined bridging hydroxyls with characteristic $\mathrm{O}-\mathrm{H}$ stretching frequencies and NMR signals; (4) In the absence of water ion-exchanged cations can take crystallographically well-defined positions in the zeolite. The transition metal ions (TMI) in these sites may be coordinatively unsaturated and can switch between oxidation states enabling redox catalysis. The large variety of available zeolite frameworks allows fine-tuning of the BAS or TMI active sites for catalysis, ${ }^{1}$ somewhat reminiscent of enzymatic active site pockets. ${ }^{2}$

Nevertheless, TMI-zeolites are complex catalysts. They tend to contain imperfections (crystal defects, silanol groups, extraframework aluminium) and mixtures of cations (commonly $\mathrm{H}^{+}, \mathrm{TMI}_{\text {, }}$ $\left.\mathrm{Na}^{+}, \mathrm{K}^{+}, \mathrm{Ca}^{2+}, \mathrm{Mg}^{2+}\right)$. Moreover the crystallographic distribution of aluminium is sparsely looked at in most studies and remains difficult to evaluate. ${ }^{3-5}$ As a consequence experimental studies on zeolite catalysts can be difficult to reproduce, comparison of data published by different research groups is challenging, and the molecular structure of active site(s) and their role in the catalytic mechanism is difficult to discern. This is particularly true for Fe-zeolites whose preparation is hampered by the aqueous chemistry of Fe cations. ${ }^{6}$

\footnotetext{
a. Department of Microbial and Molecular Systems, KU Leuven, 3001 Heverlee, Belgium.E-mail: bert.sels@kuleuven.be \& robert.schoonheydt@kuleuven.be bepartment of Chemistry, Stanford University, Stanford, California 94305, United States. E-mail: edward.solomon@stanford.edu

Department of Chemistry, KU Leuven, 3001 Heverlee, Belgium. E-mail: kristin.pierloot@kuleuven.be
}

$\mathrm{Fe}^{3+}$ is a mono-atomic aqueous complex, $\mathrm{Fe}\left(\mathrm{H}_{2} \mathrm{O}\right)_{6}{ }^{3+}$ only at $\mathrm{pH}<2$. This has two consequences: its ion exchange is invariably accompanied by ion exchange of protons, forming BAS, and as the $\mathrm{pH}$ in the pore system is unknown, oligomeric $\mathrm{Fe}_{\mathrm{x}} \mathrm{O}_{\mathrm{y}}$ nanoparticles can be formed. Alternative methods of $\mathrm{Fe}$ loading have been explored, but they all have their advantages and disadvantages and have unpredictable, complex outcomes on generally ill-defined starting materials. Advanced spectroscopic and analytic techniques are available, but currently there is no one technique that can disentangle the complexity of the catalysts as they are used in applications, especially in operating conditions.

Understanding Fe-zeolites however would be rewarding. They are applied commercially for the decomposition of $\mathrm{N}_{2} \mathrm{O}$ from nitric acid plants. ${ }^{7}$ They are researched for hydrocarbon- and ammonia assisted selective catalytic reduction of $\mathrm{NO}_{x}$ ( $\mathrm{HC}-\mathrm{SCR}$ and $\mathrm{NH}_{3}-\mathrm{SCR}$ ), performing better than currently used Cu-zeolites at high temperatures, $8,9,18-21,10-17$ and they show promise as partial and full oxidation catalysts for (unreactive) hydrocarbons with $\mathrm{N}_{2} \mathrm{O}$ or $\mathrm{H}_{2} \mathrm{O}_{2}$. Especially partial oxidation of methane to methanol and benzene to phenol are intensively studied. ${ }^{22-25} \mathrm{Fe}$ zeolites are also emerging as catalysts for the decomposition of environmental pollutants (e.g. $\mathrm{NH}_{3}$, particulates, ....). ${ }^{26-30}$

To further disentangle the complexity of Fe-zeolite catalysts beyond the state of the art, we propose a bottom-up strategy constructing the complex catalysts from well-understood building blocks. A basis of spectroscopic handles on individual catalyst components and synthesis-structure relationships is required. Simpler materials optimised for spectroscopic clarity should be studied using appropriate types of spectroscopy in carefully controlled conditions before moving on to actual catalysis in operating conditions. We first present the bottom-up approach, outlined in scheme 1 . We then substantiate the premise that the studied Fe-zeolite catalysts are often complex materials and that their characterisation is difficult. We give examples where this has led the research astray. Currently the only well understood active site in Fe zeolite catalysis is the $\alpha$ $\mathrm{Fe} / \alpha-\mathrm{O}$ site for the low temperature partial oxidation of methane and benzene with $\mathrm{N}_{2} \mathrm{O}^{31}$ and we will use this characterisation as an example paralleling the approach outlined in scheme 1 . 


\section{The Bottom-up Approach}

1) Preparation and Study of the model Fe-zeolite

A. Screen for interesting Fe-zeolites

B. Establish boundary conditions for synthesis

C. Identify spectroscopy - reactivity relationships

D. Site-selective spectroscopy \& experimentally validated models

E. Optimisation for spectroscopy

F. Full identification with (non-site-selective) techniques

G. Pinpoint full structural model

A. In situ \& operando (site-selective) spectroscopy on intermediates

B. Identify reaction pathways in individual reaction steps

C. Identify engineering opportunities

3) Assemble improved functional catalysts

A. Assemble optimised components into an optimal catalyst

B. Test catalytic performance in realistic conditions

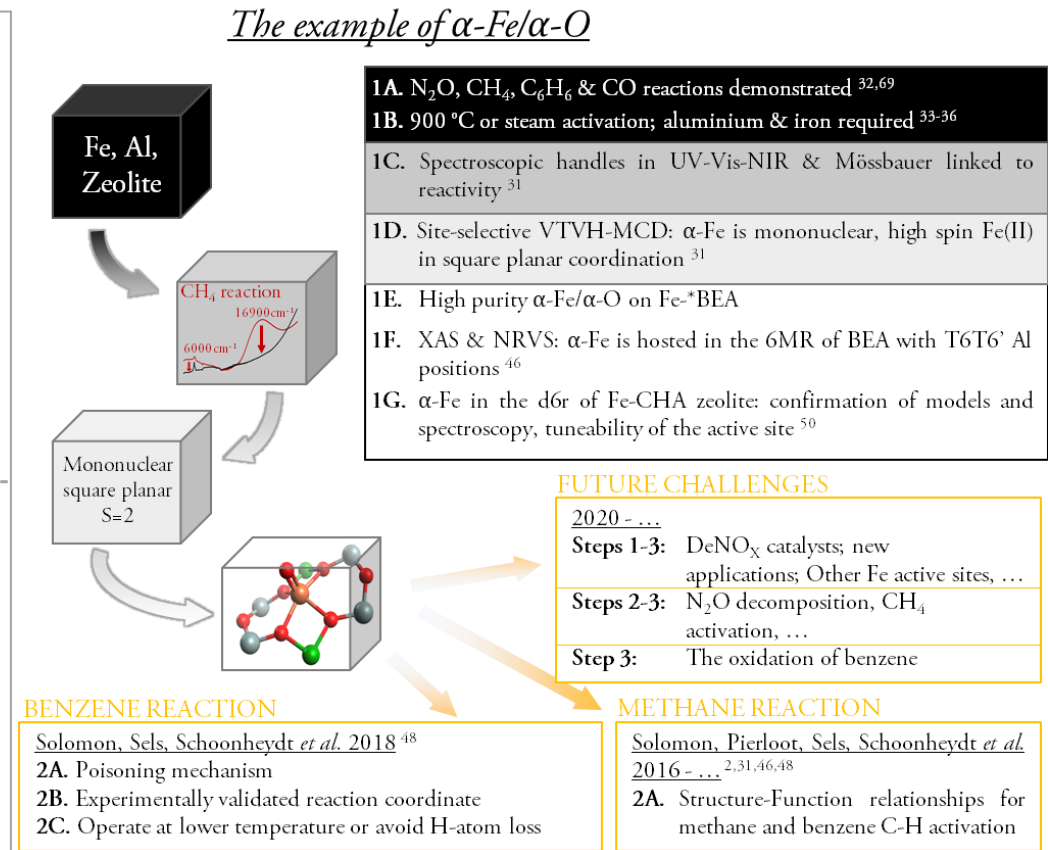

Scheme 1: Left: Sequential methodology for understanding TMI zeolite catalysts; Right: The methodology as applied for the identification of the $\alpha$-Fe/ $\alpha$-O active site

\section{Discussion}

\section{A bottom-up approach to understand Fe-zeolite catalysis}

Building a foundation for Fe-zeolites. A step-by-step outline to achieve a robust, long term understanding of Fe-zeolite catalysis is given by the three steps shown in the frame of scheme 1. First, a material of interest should be identified and prepared reliably, usually in relation to some measure of catalytic performance (steps $1 \mathrm{~A} \& \mathrm{~B}$ ). Then spectroscopic probes should be identified to allow tracking of the active site (step 1C). Using site-selective spectroscopy the Fe site(s) of interest can be characterised among less interesting iron (step 1D). These data can be used to support spectroscopically validated molecular models leading to some initial structural understanding. From this a more active-site selective synthesis method can be set up for each Fe site of interest (step 1E). Having prepared Fe-zeolites with high loadings of the interesting Fe site, or with an interesting Fe site among other well described Fe sites then allows further refinement of the structural identification and reliable reactivity tests directly relatable to the $\mathrm{Fe}$ in the sample (step $1 \mathrm{~F}$ ). Note that changes to the synthesis will affect the Fe speciation, therefore steps $1 \mathrm{~A}-1 \mathrm{G}$ are marked 'material specific' in scheme 1. The spectroscopic features and iron speciation may also differ in different zeolites with different aluminium distributions, pore systems, cages, defects, etc. Therefore some iterations may be needed in the first steps.

Diffuse reflectance UV-Vis (DR-UV-Vis) and Mössbauer spectroscopy (with ${ }^{57} \mathrm{Fe}$ ) are commonly used to study iron sites. These are spectroscopic methods that measure all of the iron in a sample but reveal resolvable features from individual iron species. By correlating these data with reactivity, specific spectroscopic features can be assigned to an active iron site. A specific absorbance feature can then be probed with site-selective spectroscopies. These include resonance Raman ( $r$ R) spectroscopy to gain vibrational insight and variable-temperature variable-field magnetic circular dichroism (VTVH-MCD) to elucidate the electronic structure of an active site. These methods are powerful because they can be tuned into a specific absorbance feature and thus be used to study a specific site in zeolites with multiple iron sites present that do not participate in the reaction. Once a Mössbauer signal correlates quantitatively with reactivity it can be fit to obtain geometric and electronic structural information about this Fe site (spin state, redox state and coordination environment). The assignment of specific spectral features to an active site also allows the synthesis of the sample to be monitored and tuned to maximize the amount of active iron in the sample. This then allows bulk structural techniques to be used that include nuclear resonance vibrational spectroscopy (NRVS) which gives vibrational data on all of the iron in a sample and X-ray absorption spectroscopy (XAS) which gives structural information on all the iron. Unlike DR-UV-Vis and Mössbauer spectroscopy, these methods do not give resolvable features for the separate Fe sites present, but once the active site's concentration is maximized and the nature and spectroscopic contributions of any remaining Fe are determined they provide unique geometric and electronic structural insight.

Once a well-defined starting point is obtained, we can move on to step 2 in the scheme, bringing the Fe-zeolites in contact with reagents one by one. When these interactions are understood, the same can be done building up to realistic reaction conditions. Finally, knowing the active Fe species and their interactions with relevant molecules under the required conditions, the sub-reactions identified in step 2 are brought together in step 3. As such, performant catalysts are designed that perform a by-design mechanism made up of the sub-reactions investigated in step 2 .

\section{Three decades uncovering the $\alpha-\mathrm{Fe} / \alpha-\mathrm{O}$ sites}

The activation of benzene and methane with $\mathrm{N}_{2} \mathrm{O}$ over Fe-zeolites is known for over 30 years, but the identification of the active site and intermediates has been achieved only recently. Initially, the selective benzene to phenol reaction was attributed to Brönsted acid catalysis, ${ }^{32}$ until Panov et al. found a correlation with $\mathrm{Fe}$, which could be extrapolated to Fe impurities in the original H-ZSM-5 materials. ${ }^{33}$ They then coined the active site $\alpha-F e$, forming the reactive 
intermediate named $\alpha-\mathrm{O}$ from $\mathrm{N}_{2} \mathrm{O} .{ }^{34}$ Based on Mössbauer spectroscopy, reactivity data, and the alluring comparison to the active site in the soluble methane mono-oxygenase enzyme, their initial active site assignment was an Fe(III) dimer.6, 23,35 Panov et al. did valuable work establishing synthesis methods to prepare active materials, identifying activation at $900^{\circ} \mathrm{C}$ or in steam at $550^{\circ} \mathrm{C}$ as important steps to improve the active site density. ${ }^{35,36}$ In such steps some aluminium is known to leave the zeolite framework and this was by some authors immediately translated into a new active site proposal - a spinel of aluminium and iron oxide. ${ }^{37}$ While this was a reasonable hypothesis, it lacked spectroscopic evidence and appropriate experimental methods to prove such structures essential for activity. The formation of extra-framework aluminium has turned out to likely be a confounding variable in the conversion of iron into $\alpha$-Fe active sites (see later). The misinterpretations can be attributed to the omission of steps $1 \mathrm{C}-1 \mathrm{G}$ in scheme 1.

Many studies have used a combination of UV-Vis and Mössbauer spectroscopy to look for Fe active sites, and while previous studies had observed the small quadrupole split doublet that was later assigned to $\alpha-\mathrm{Fe}$, this was never correlated with reactivity and was not identified as a spectroscopic handle of the active site. ${ }^{38,39}$ Without coupling these spectroscopies to reactivity in materials with heterogeneous iron species, both techniques lose their power and it becomes difficult to sort through the unknown signals Deconvolution becomes unreliable, especially for quantification. Consequently the techniques cannot be used to probe active sites with sufficient selectivity. Difference spectra and modulation spectroscopic methods may help, but often don't sufficiently remove the complexity of the spectra. Especially inappropriate are sample averaging techniques such as EXAFS and standalone reactivity data. Pirngruber et al. have demonstrated the need to triangulate multiple spectroscopic techniques to avoid incorrect assignments of dimeric iron species in Fe-ZSM-5, showing a combination of EXAFS, UV-Vis and MCD was needed. ${ }^{40}$ Characterisation of active sites requires their confirmation through reactivity testing (step $1 \mathrm{C}$ ). This was omitted in a RIXS study on Fe-zeolites leading to the incorrect conclusion that "the formation of Fe(IV) upon reaction of Fe-ZSM-5 with $\mathrm{N}_{2} \mathrm{O}$ can be ruled out". ${ }^{41} \mathrm{MCD}$ data later on did demonstrate an $\mathrm{Fe}(\mathrm{IV})$ site was in fact correlated with the room temperature reaction with $\mathrm{CH}_{4}$ to form $\mathrm{CH}_{3} \mathrm{OH} .{ }^{31}$ The RIXS study used a single preparation method of Fe-ZSM-5 from Fe(II) $\mathrm{Cl}_{2}$, not representative for Fe-ZSM-5 in general. Other preparations from Fe(II) are also known to yield little $\alpha$-Fe sites. ${ }^{42}$ In addition, there were no tests for methane or benzene activation.

To circumvent the experimental challenges on $\alpha-\mathrm{Fe} / \alpha-\mathrm{O}$, numerous purely computational studies have been published. In hindsight, some of these were on the right track, ${ }^{43}$ while others were far off With a limited number of boundary conditions set by experiment, modelling at sufficiently high levels of theory can provide quite accurate predictions on active site geometry and electronic structure. ${ }^{44}$ Between a restricted number of possible configurations, the condition dependence of different Fe species' stability in zeolites can also be predicted with reasonable confidence. ${ }^{45}$ The information from these studies, whether relevant to $\alpha-\mathrm{Fe} / \alpha-\mathrm{O}$ or not, may give useful insight in $\mathrm{Fe}$ sites for catalysis when they become experimentally defined in the future. Question remains if this is an efficient approach to the problem. Contrary to experimental screening, accurate prediction and optimisation of Fe active sites for catalysis remain cumbersome in silico. At the levels of model simplicity that enable broad screening of distinct active site structures, errors in predicted activation energies tend to exceed what is needed for useful predictive power of catalytic performance.

Towards a full characterisation of $\alpha-\mathrm{Fe} / \alpha-0$ sites. In 2016 an iron site reacting with $\mathrm{N}_{2} \mathrm{O}$ to form an intermediate with identical reactivity and other properties to that of Panov's $\alpha-O$ was described on the Fe-*BEA zeolite and could thus be safely designated as $\alpha$-Fe on ${ }^{*}$ BEA. ${ }^{31}$ This was a key step in optimising the material for spectroscopy. While $\alpha$-Fe on Fe-*BEA closely parallels that on FeZSM-5 and Fe-FER both in reactivity and spectroscopy, it has two key advantages. Its $\alpha$-Fe site's $d_{z^{2}}-d_{x^{2}-y^{2}}$ absorption band is uniquely intense and it hosts mostly a single-site $\alpha-\mathrm{Fe}$ at low loadings. Especially the strong $d_{z^{2}-} d_{x^{2}-y^{2}}$ band facilitated crucial VTVH-MCD experiments. Because the spectroscopic features identified on $\mathrm{Fe}$ *BEA translate to Fe-MFI, the data on Fe-*BEA facilitate the study of the spectroscopically less accessible Fe-MFI active sites as well.

Using VTVH-MCD, UV-Vis-NIR and Mössbauer spectroscopy, the $\alpha$-Fe site on Fe-*BEA zeolite was identified as a mononuclear, high-spin $\mathrm{Fe}(\mathrm{II})$ bound with square planar coordination in an exchange position of the zeolite. ${ }^{31,46}$ The $\alpha-0$ site was identified as a square pyramidal, high-spin $\mathrm{Fe}(\mathrm{IV})$ with a terminal oxo ligand added to its $\alpha$-Fe precursor. $31,46,47$ The steps of their identification have broadly followed those discussed in the previous section and are shown on the right-hand side of scheme 1 . Most of the characterisation work (steps 1A-1D) has been reviewed at length in a recent review, ${ }^{2}$ as has the reaction with methane to some extent (step 2A). Here we will focus on the additional work that has been done since, $46,48-50$ covering steps $1 \mathrm{E}-1 \mathrm{~F}$ for the $\alpha-\mathrm{Fe} / \alpha-\mathrm{O}$ sites in general, and steps $2 \mathrm{~A}$ $2 \mathrm{C}$ for the benzene reaction specifically.

Most of this work has been done on Fe-*BEA and Fe-CHA zeolites, and not on Fe-ZSM- 5 on which $\alpha$-Fe was first defined by its capability of forming a reactive surface-bound oxygen $(\alpha-O)$ from $\mathrm{N}_{2} \mathrm{O}$, that reacts with methane and benzene at room temperature. However, this definition may in theory encompass multiple types of active sites as long as they satisfy the reactivity conditions, and it is unnecessary to restrict this to only certain zeolite topologies, or to certain reaction conditions. We propose that the structural description established in refs 31,46 and 50 presents a more appropriate definition, rooting in more fundamental characteristics of the active site.

EXAFS and NRVS on $\alpha-F e$ and $\alpha-0$ [1E \& 1F]. The characteristic Mössbauer doublet and intense high energy $d_{z^{2}}-d_{x^{2}-y^{2}}$ absorption band of $\alpha-\mathrm{Fe}$ on Fe-*BEA were used to maximise the amount of the $\alpha$ $\mathrm{Fe} / \alpha-\mathrm{O}$ site in the zeolite. Samples were optimised to contain $>70 \%$ of iron as $\alpha-\mathrm{Fe}$ or $\alpha-\mathrm{O}$ which made it possible to use the powerful but bulk spectroscopies, NRVS and XAS, to gain geometric insight into the active site. ${ }^{46}$ The ability to correlate reactivity with spectroscopy to maximise the amount of active site in the samples was a significant break-through in allowing new spectroscopic methods - in particular NRVS - to be accurately used. Using bulk spectroscopies without this important first step, as is often the case in heterogeneous systems, would produce signals that reflect the average of all the iron in the sample, limiting the effectiveness and accuracy of these techniques. For EXAFS in particular, active site purities of $>75 \%$ were required to reliably identify the scattering path associated with the $\mathrm{Fe}=\mathrm{O}$ bond of $\alpha-O$. This technique is therefore highly susceptible to interference from spectator sites.

Coupling the NRVS vibrational data to density functional theory (DFT) models and XAS data the geometric structure of $\alpha-\mathrm{Fe}$ in *BEA was 
revealed as an $\mathrm{Fe}(\mathrm{II})$ square planar site in a $\beta$-type 6 membered-ring (MR) with two aluminium atoms located at T6 lattice tetrahedral positions (or analogous positions in other $\beta$-type 6MRs). This $\alpha$-Fe geometric assignment reinforced the earlier VTVH-MCD data. The > $70 \% \alpha$-Fe sample was converted to $\alpha-\mathrm{O}$ by reaction with $\mathrm{N}_{2} \mathrm{O}$, allowing the geometric structure of $\alpha-O$ to be defined with this combination of NRVS and XAS as well. The $\alpha-O$ site was located in the same $\beta-6 \mathrm{MR}$ as the $\alpha-\mathrm{Fe}(\mathrm{II})$ precursor, but with an additional axial oxygen atom bound to the Fe creating a square pyramidal structure. The Fe-O bond was found to be the strongest ever identified, as indicated by a uniquely high energy Fe-O stretch at $885 \mathrm{~cm}^{-1}$ The absence of a trans axial ligand helped explain $\alpha-\mathrm{O}^{\prime} \mathrm{s}$ high reactivity. This assignment also supported the $\mathrm{Fe}(\mathrm{IV})=0$ square pyramidal $\mathrm{S}=2$ definition of the active site previously determined using VTVH-MCD and agreed with computational models based on the previous data.

$\alpha-\mathrm{Fe} / \alpha-\mathrm{O}$ on CHA versus *BEA [1G leading to $2 \mathrm{C}$ ]. The NRVS and XAS data showed $\alpha$-Fe in the *BEA zeolite is hosted in a 6MR with two aluminium substitutions at opposite sides of the 6MR. Motivated by its high density of $6 \mathrm{MRs}$ and relatively simple pore structure, Fe-CHA was therefore investigated next. Fe-CHA was shown to host $\alpha-\mathrm{Fe}$ and $\alpha-\mathrm{O}$ sites capable of $\mathrm{CH}_{4}$ activation in its $\mathrm{d} 6 \mathrm{r}$ structural building blocks, and it could also host single site $\alpha$-Fe at low iron loadings. ${ }^{50}$ The electronic absorption spectrum of $\alpha-\mathrm{Fe}$ in $\mathrm{CHA}$ differed predictably from that of $\alpha$-Fe in *BEA given the more symmetric and wider $6 \mathrm{MR}$ of $\mathrm{CHA}$ (figure 1), lending further experimental support to the proposed $\alpha$-Fe models. The wider 6 MR brings the $d_{z^{2}}-d_{x^{2}-y^{2}}$ transition down from 15900 to $13000 \mathrm{~cm}^{-1}$ and the higher symmetry results in a lower extinction coefficient of the high energy $d-d$ transition relative to the lower energy $\mathrm{d}$ - $d$ transition at $5000 \mathrm{~cm}^{-1}$. While this means some of the ground work of active site characterisation will need to be redone for each new support material, it also indicates that its properties will increasingly be predictable with in silico models. Moreover, interpretation of the spectroscopy will become increasingly straightforward with each new material and the full range of techniques will not be required.

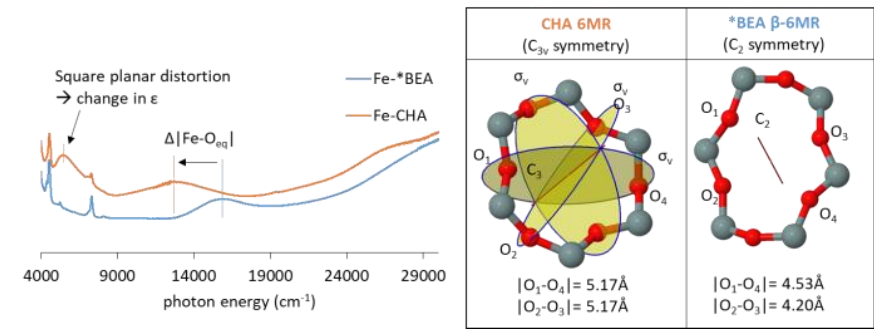

Figure 1: Left: electronic absorption spectra of single-site $\alpha$-Fe on Fe-*BEA and Fe-CHA Right: The 6MRs found in *BEA and CHA that host $\alpha$-Fe. ${ }^{50}$

Expanding the spectroscopic basis of the $\alpha-\mathrm{Fe} / \alpha-\mathrm{O}$ sites to other zeolite topologies will enable tuning of the active site's confinement and accessibility, and of the iron's ligand field (both in strength and in symmetry). Once a topology is available per variable, unambiguous experimental probing of these properties on catalysis will for the first time be possible. Work of this type has been demonstrated on the $\mu$ oxo bridged dicopper active sites in ZSM- 5 and MOR, where the zeolite environment around the active site affected the methane $\mathrm{H}$ abstraction activation energy. ${ }^{51,52}$ On Fe zeolites computational studies have explored the effect of confinement, but starting from unconfirmed active site structures these are of questionable validity to real catalysis, and should be revisited. ${ }^{53-55}$ Knowing the effects of the mentioned zeolite and active site properties on specific reactions will enable step $2 \mathrm{C}$ in scheme 1, engineering the right Fe-zeolite for specific applications.

The benzene reaction: a bottom-up reaction mechanism [2A-2C]. Detailed evaluation of the $\alpha-\mathrm{Fe}$ and $\alpha-O$ species (vide supra) provided the foundation to determine the mechanism of catalytic benzene hydroxylation by Fe-zeolites. This reaction is notable as the key step of the AlphOx process, which was tested at the pilot plant level. ${ }^{56}$ While this process is remarkably selective for the phenol product, it was ultimately abandoned due to issues with catalyst deactivation via coke formation.

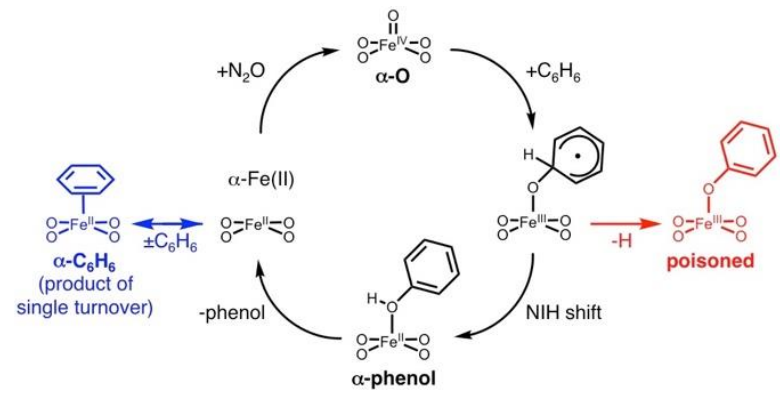

Figure 2. Cycle for benzene hydroxylation to phenol catalysed by $\alpha$-Fe. The product of single turnover, $\alpha-\mathrm{C}_{6} \mathrm{H}_{6}$, is indicated in blue. The phenolate poisoned active site, generated in small quantities during single turnover, is indicated in red. ${ }^{48}$

The single turnover reaction of $\alpha-O$ with benzene was evaluated, using Mössbauer spectroscopy as a quantitative probe of $\mathrm{Fe}$ speciation. It was determined that $\alpha-O$ converts nearly quantitatively into a high spin ( $\mathrm{S}=2) \mathrm{Fe}(\mathrm{II})$ site $\left(\alpha-\mathrm{C}_{6} \mathrm{H}_{6}\right.$, blue species in figure 2$)$ that is distinct from $\alpha-\mathrm{Fe}(\mathrm{II})$. A combination of Fe K-edge XAS and ${ }^{57} \mathrm{Fe}$ NRVS showed the single turnover product is the substrate-trapped active site. Thus over the course of single turnover, the phenol product is released from the active site, regenerating $\alpha$-Fe, and closing the catalytic cycle. ${ }^{48}$

This result was at odds with past studies of the single turnover, which focused on a $13900 \mathrm{~cm}^{-1}$ absorption band that appears upon the reaction of $\alpha-\mathrm{O}$ with $\mathrm{C}_{6} \mathrm{H}_{6} .{ }^{57}$ Raman studies of the reacted catalyst, using laser excitation within the absorption envelope, revealed a number of vibrations $\left(v(\mathrm{Fe}-\mathrm{O})=643 \mathrm{~cm}^{-1}\right.$ among others) consistent with a phenolate-ligated species. On this basis, the main product of single turnover was mistakenly identified as a phenolate-bound diferric species. A combination of resonance Raman spectroscopy and $M C D$ spectroscopy later enabled the correct assignment of the chromophore as a mononuclear $\mathrm{S}=5 / 2 \mathrm{Fe}(\mathrm{III})$-phenolate that forms at the active site (figure 1 , red species). ${ }^{48}$ Mössbauer spectroscopy identified this as a minority species, representing only $<5 \%$ of Fe next to the majority $\alpha-C_{6} H_{6}$ product. The $\mathrm{Fe}(\mathrm{III})$-phenolate is a poisoned state of the active site, and a likely precursor to the formation of hard aromatic coke, and thus catalyst deactivation.

Thus, multispectral study of the single turnover reaction revealed two competing mechanisms: a productive catalytic cycle producing phenol, and an irreversible deactivating reaction producing $\mathrm{Fe}(\mathrm{III})$ phenolate. DFT models, calibrated against spectroscopic data and experimental H/D kinetic isotope effects, provided detailed insight into these mechanisms. First, $\alpha-O$ attacks benzene, oxidizing the substrate by one electron and forming a new $\mathrm{C}-\mathrm{O}$ bond. While $\mathrm{C}-\mathrm{O}$ bond formation is typically rate limiting in aromatic hydroxylation 
reactions, $\alpha-\mathrm{O}$ is so highly activated for electrophilic chemistry that this step proceeds without an activation barrier. The resulting $\alpha$ complex contains an $\mathrm{S}=5 / 2 \mathrm{Fe}(\mathrm{III})$ centre antiferromagnetically coupled to a substrate radical. The fate of the substrate - phenol or phenolate - is determined by the fate of the ipso $\mathrm{C}-\mathrm{H}$ bond. This bond is extremely weak, and can cleave homolytically to form an Fe(III)phenolate with a modest barrier. However, there is a lower barrier process in which the ipso proton migrates to the ortho carbon (NIH shift), triggering reduction of the $\mathrm{Fe}$ centre, and forming cyclohexadienone. The dienone then tautomerizes to the phenol product. $^{48}$

Experimental data coupled to DFT simulations provided key insight into how Fe-zeolites catalyse the selective hydroxylation of benzene to phenol at high levels of substrate conversion. Because this reaction was determined to be diffusion limited (or involving a very low barrier), selectivity is governed by the relative diffusion rates of benzene and phenol through the zeolite lattice. The hydrophilic phenol product interacts strongly with zeolite lattices, and as a result it reacts sluggishly despite its much higher intrinsic activation toward electrophiles. High residence of phenol in the zeolite opens routes to undesired secondary products. Moving forward, it will be important to better understand how the zeolite lattice structure tunes the branching ratio between phenol production and phenolate poisoning. This Fe-zeolite chemistry may also provide a useful mode for benzene hydroxylation by $\mathrm{Cu}$-zeolites, ${ }^{58}$ which make use of $\mathrm{O}_{2}$ as an oxidant, but potentially proceed through a more complicated (and currently unknown) mechanism.

\section{Future challenges in $\alpha-\mathrm{Fe} / \alpha-0$ zeolite catalysis}

The proposed bottom-up methodology requires a systematic study of individual parameters, repeated again for each application. The number of possible experiments grows exponentially with the number of parameters. Of course a lot can already be learnt from trends identified in the available literature. Supplemented with common sense these can help us prioritise.

Some follow-up steps were already mentioned on tuning the Fezeolites by varying zeolite topology and on further development of Fe-zeolites for the conversion of benzene. The bottom right box of scheme 1 suggests some other paths to valorise the work on characterising the $\alpha-\mathrm{Fe} / \alpha-\mathrm{O}$ active sites. Given the site's extraordinary reactivity and its preferential formation on many $6 \mathrm{MR}$ zeolites $^{31,44,50}$ it may well have played a role in a lot of the Fe-zeolite catalysis studied in the past, even if $\alpha-\mathrm{Fe} / \alpha-\mathrm{O}$ was not detected. Fezeolites are intensively investigated for $\mathrm{SCR}^{8-21,59}$ and the activity of isolated Fe and $\alpha-\mathrm{Fe}$ on ZSM- 5 zeolite has often been proposed. ${ }^{12}$, 14,15,60,61 Therefore the site's role in DeNOx mechanisms should be clarified. Also reactions that are well known to be catalysed by $\alpha$ $\mathrm{Fe} / \alpha-\mathrm{O}$ sites such as $\mathrm{N}_{2} \mathrm{O}$ decomposition and methane oxidation should now be investigated further on a molecular level. For the former reaction the interaction of $\mathrm{N}_{2} \mathrm{O}$ with $\mathrm{Fe}$ and the recombination of $\alpha-\mathrm{O}$ to $\mathrm{O}_{2}$ should be clarified, and on a longer term also the effect of confinement, active site separation, Brönsted acid sites (BAS) and other TMI cofactors. Some of these topics have been researched before, ${ }^{35,} 38,54$ but never on single active site Fe-zeolites and never with full knowledge of the active site structure or its spectroscopic handles. For the latter reaction $\left(\alpha-\mathrm{O}+\mathrm{CH}_{4}\right)$, currently investigated $\alpha-\mathrm{Fe}$ zeolites span already a few topologies (FER, CHA $\left.\mathrm{ZSM}-5,{ }^{*} \mathrm{BEA}\right)^{31,38,50}$ but methane conversion remains stoichiometric with low single cycle yields (highest reported is $70 \mu \mathrm{mol} / \mathrm{g}$ ). ${ }^{62}$ Moving forward, the effect of counter-ions (BAS, other TMI, $\mathrm{Na}^{+}, \ldots$ ) and the pore system on enabling high selectivity and (quasi-)catalysis as opposed to stoichiometric reaction should be looked into. $\mathrm{Fe}-\mathrm{OCH}_{3}$ and $\mathrm{Fe}-\mathrm{OH}$ have been suggested from bulk vibrational data after the reaction of $\alpha-\mathrm{O}$ with $\mathrm{CH}_{4},{ }^{63-67}$ but it is not known how this relates to the $\alpha$-Fe active sites. Variable turnover numbers (TON) for the reaction have been reported on different Fe-ZSM-5 preparations, ${ }^{62,66-69}$ but the underlying mechanism is unclear. The effect may be related to the pore system, to the Fe speciation, to the $\alpha$-Fe ligand field, or a combination of factors. Besides (and related to) the low TON, the recovery of products remains a challenge. 50,62 To recover methanol, it has been proposed that Fe-methoxy must be released through the addition of water. Water adsorbs strongly on $\alpha-\mathrm{Fe}$, and on zeolites in general, and its presence hampers regeneration of $\alpha-O$ and thus obstructs higher TON. Brought together, these studies should provide new solutions to break through the common conversion - selectivity trade-off typical for partial oxidation reactions, enable higher TON, and provide catalytic mechanisms with reasonable methods of product - and active site recovery.

Another way to improve reactivity is increasing the concentration of $\alpha-\mathrm{Fe}$. A lot of work has been done on this in the past, $35,36,70$ but again without a clear handle on $\alpha-\mathrm{Fe}$ or $\alpha-\mathrm{O}$. Quantification was often based on the oxygen abstraction from $\mathrm{N}_{2} \mathrm{O}$, but that may not be a sufficiently discriminating measure to identify $\alpha-\mathrm{Fe} .{ }^{71}$ While many variables have been tested to optimise the $\alpha$-Fe concentration, especially on $\mathrm{MFI}$, the exact mechanism of its formation in various preparation methods remains largely unknown. If this is understood, a more rational design of synthesis recipes to obtain $\alpha$-Fe both in high concentration and in high purity on zeolites becomes possible. With these improved materials better catalysis becomes possible, but also more detailed mechanistic studies. We now know that $\alpha$-Fe is located in 6MRs with two oppositely positioned aluminium substitutions (e.g. the T6 positions in a $\beta-6 \mathrm{MR}$ of *BEA lattice based on NRVS data). ${ }^{31,46,50}$ Knowing the structural requirements we can identify suitable support materials and effective methods of $\mathrm{Fe}$ introduction.

To further explore the role of $\alpha-\mathrm{Fe} / \alpha-\mathrm{O}$ type sites in current $\mathrm{Fe}$ zeolite catalysis, their behaviour in typical reaction conditions and after aging should be investigated. Mobilization of TMIs in conditions of automotive exhaust equipped with $\mathrm{NH}_{3}$-SCR systems is known. ${ }^{72-}$ ${ }^{74}$ Under such conditions $\alpha$-Fe sites may reassemble to form strongly ligated $\mathrm{Fe}$ in the pore system, or may aggregate into multimeric species. ${ }^{75}$ In aqueous solutions Fe catalysis is investigated for Fenton reactions and the low temperature activation of methane with $\mathrm{H}_{2} \mathrm{O}_{2} \cdot{ }^{30,76-78} \mathrm{Also}$ gas-phase catalysis will commonly involve some percentages of water vapour. The interaction of $\alpha$-Fe and $\alpha-O$ with $\mathrm{H}_{2} \mathrm{O}$ was investigated before by Panov et al. ${ }^{79}$ Again, no spectroscopic handles on the active sites were available at the time and it will be worthwhile revisiting this work. As in the SCR conditions, it will be interesting to test whether $\alpha$-Fe reassembles in aqueous conditions or largely retains its structure and is also catalytically active in these conditions for certain reactions.

\section{Probing other monomeric and dimeric Fe sites in zeolites}

The $\alpha-\mathrm{Fe} / \alpha-\mathrm{O}$ active site is monomeric. The active site cycles between +2 and +4 oxidation states, ${ }^{31}$ and is unlikely to effectively form the typical reactive oxo intermediates from $\mathrm{O}_{2}$, requiring a four electron reduction. Many homogeneous and enzymatic Fe catalysts 
contain Fe dimers, ${ }^{80}$ which have also been proposed on Fe-zeolites Dimeric active sites may exchange more electrons enabling full $\mathrm{O}_{2}$ reduction. However, a definite spectroscopic identification of such dimers on zeolites remains elusive. ${ }^{40} \mathrm{Hammond}$ et al. have proposed an $\mathrm{Fe}-(\mathrm{OH})_{2}-\mathrm{Fe}$ active site catalysing methane oxidation with $\mathrm{H}_{2} \mathrm{O}_{2}$ based on $\mathrm{rR}$ tuning into a $33000 \mathrm{~cm}^{-1}$ absorption band which definitely merits further probing. ${ }^{81}$ In their system methane oxidation is catalytic and maintains high selectivity, although conversion and mass based turnover frequency remain low. It must be said that the characterisation of larger Fe species will likely be much more difficult than that of $\alpha$-Fe. The $\alpha-F e$ can relatively easily be formed as a single site and provides unique spectroscopic handles in electronic absorption and Mössbauer spectroscopy. ${ }^{31,50}$ As with copper, iron dimers will likely only be formed together with other iron species, not as a single site. ${ }^{6}$ Again, gradually building up complexity may be the answer. Moving up in Fe loading from the single-site $\alpha$-Fe materials, Fe spectators appear in the UV-Vis and Mössbauer spectra of Fe-*BEA. ${ }^{31}$ These are likely monomers in the other exchange sites of the zeolite, as calculated by Hallaert et al. ${ }^{44}$ Their exact coordination environment should be confirmed with site selective techniques (VTVH-MCD, rR). While these may be less relevant for catalysis, taking a step back to figure out the less glamorous spectroscopy will pay off eventually as they will likely cloud the spectroscopy of dimers that should start forming upwards a certain Fe/Al ratio. Dimers may also occur in more configurations than monomers can. The Fe ions can be bridged by various numbers of $\mathrm{OH}$ or $\mathrm{O}$ ligands, and these bridges may be sensitive to conditions. To our knowledge, the only other fully characterised Fe in zeolites is tetrahedral high-spin Fe(III) that is incorporated in the zeolite framework by isomorphic substitution. This site is, like $\alpha-\mathrm{Fe}$, also mononuclear and can also be synthesised as a single-site. ${ }^{82-87}$ Clusters of Fe(III) oxides are also fairly well defined spectroscopically, but the standards for their 'full' characterisation are generally lower. Distinction between clusters of similar composition is equally difficult but often less important.

For Fe(II) sites, both mononuclear and binuclear species will have $\mathrm{d}$ $d$ transitions at similar energies in the NIR, however these can be distinguished by VTVH MCD as the binuclear Fe(II) sites will undergo additional exchange coupling associated with the bridging ligation. VTVH-MCD data reflect this coupling, and can therefore probe the nature of the bridge. For $\mathrm{Fe}(\mathrm{III})$ sites, $d$ - $d$ transitions are spinforbidden and therefore tend to not be observed. On the other hand $\mathrm{Fe}$ (III) species in zeolites often have LMCT transitions in the UV-vis region. While these transitions can be broad and overlapping, they still can be probed selectively using VTVH-MCD (vide supra) or $\mathrm{rR}$ spectroscopy. As Fe(III) dimers in zeolites are liable to be strongly antiferromagnetically coupled through bridging $\mathrm{O}(\mathrm{H})$ ligands, however, rR will likely be a more useful technique. By using a range of laser excitation energies spread out over a dimer absorption band of interest, it is possible to identify the characteristic Raman vibrations of the chromophore. A key advantage of $r R$ is its sensitivity to the identity of bridging ligands, especially when coupled to isotopic perturbation. The bridging ligands are often intimately related with reactivity. Finally, Mössbauer spectroscopy provides a convenient mechanism to distinguish monoferric and diferric sites. In the presence of a small external magnetic field, monoferric species will contribute hyperfine-split six-line patterns, reflecting the ground state paramagnetism of the isolated ferric ion. Diferric species wil likely be antiferromagnetically coupled, and therefore remain diamagnetic at low temperature. These species will not display hyperfine splitting, and instead contribute two simple quadrupole doublets - one for each Fe(III) centre.

The spectroscopic challenge may alternatively be circumvented by tackling the synthesis of (approximately) single-site Fe dimers. Given that the challenge of spectroscopic identification will only get more complicated for reaction intermediates as reactions and operando conditions are introduced, improving the synthesis will be indispensable. A good starting point may be the Fe-zeolite preparation methods that are known to not yield $\alpha-\mathrm{Fe}$, even at low loadings, but may contain Fe dimers. The already mentioned method introducing ferrous precursors under inert atmosphere is the only clear example resulting in a very different iron speciation, ${ }^{41,88}$ however the iron species remain heterogeneous, so new inventive strategies will likely be needed. Getting a grip on the aluminium distribution is another requirement to get closer to single site $\mathrm{Fe}$ zeolites at higher loadings. Especially on SSZ-13 zeolites control over the siting of framework aluminium is a lively research topic. ${ }^{49,89}$ Here computational modelling can help to predict which host materials and post treatment procedures would be most suitable to achieve high densities of which types of Fe sites. Some insightful studies already exist showing the conditions in which particular $\mathrm{Fe}$ monomers and/or dimers are thermodynamically favoured. ${ }^{44,45}$

\section{Fe active sites on zeolites for $\mathrm{NH}_{3}$-SCR of $\mathrm{NO}$}

CU-SSZ-13 and CU-SAPO materials are now commercialised for automotive $\mathrm{NH}_{3}$-SCR ${ }^{90,91}$, but Fe-zeolites are known from research to potentially outperform Cu-zeolites at higher temperatures. ${ }^{60,92}$ Despite a large number of publications research has so far failed to bring notable improvements to the commercial copper catalysts in the past decade and especially the Fe catalysts remain poorly understood. ${ }^{59,91}$ Past research has identified trends between synthesis and performance, but the connecting links between the two using spectroscopy remain lacking. ${ }^{59}$ The slowdown of tangible results indicates another approach may be warranted. Compared to the low temperature oxidation of benzene and methane with $\mathrm{N}_{2} \mathrm{O}$ discussed above, $\mathrm{NH}_{3}$-SCR of $\mathrm{NO}$ entails far more complex reaction conditions, especially when realistic operating conditions are considered. ${ }^{93,94}$ The system most likely involves a complex reaction scheme with countless intermediates and multiple active sites. ${ }^{60,95}$ Every additional reagent, reaction, adsorbent, or active site may interact with every other component, rapidly increasing the complexity. Without basic knowledge of the individual components it seems unrealistic to fundamentally understand these catalysts. It may be more rewarding to take a step back and investigate the individual interactions of reaction components before returning to more complex materials and conditions. Only then will fundamental relations between catalyst properties (confinement, counter-ions, ...) and catalytic performance become accessible to experiment.

\section{Concluding remarks}

Characterisation of $\mathrm{Fe}$ active sites in zeolites remains a grand challenge that should be addressed simultaneously on the fronts of synthesis and spectroscopy. In the first research stages, synthesis should foremost target site homogeneity, leaving the challenge of catalyst optimisation for actual catalysis for a later stage when structures and mechanisms are better understood. Site selective spectroscopy has proven valuable in identifying active sites of interest, providing clearer targets for synthesis. This has been the case for $\alpha$-Fe active sites probed with MCD spectroscopy and can also become the case for the active sites in low-temperature methane 
activation with $\mathrm{H}_{2} \mathrm{O}_{2}$ probed with resonance Raman. The only thoroughly characterised catalytically active iron site in zeolites is $\alpha$ Fe and we have demonstrated how its characterisation has been achieved. The use of appropriate bulk and site-selective spectroscopies created a systematic approach to understanding this complex system. Coupling spectroscopy with synthesis to create a pure sample opened up the possibility of using bulk techniques to gain structural insight that would have been impossible with a mixture. This coupling of spectroscopy with synthesis and catalysis creates a working model on how other challenging problems can be addressed in the future. More daunting challenges will be faced in unravelling the full mechanisms of larger active sites, namely dimers, in more complex reaction conditions. While advanced techniques are available, such tasks will only be tackled through multiple controlled, systematic steps.

\section{Conflicts of interest}

There are no conflicts to declare.

\section{Acknowledgements}

Funding was provided by Research Foundation Flanders (FWO Grant G0A2216N to B.F.S., K.P. and R.A.S.) and the National Science Foundation (grant CHE-1660611 to E.I.S.). B.E.R.S. acknowledges support from the National Science Foundation Graduate Research Fellowship Program under grant DGE-11474, and from the Munger, Pollock, Reynolds, Robinson, Smith \& Yoedicke Stanford Graduate Fellowship.

\section{References}

1. Baerlocher, C. et al. Database of Zeolite Structures http://www.iza-structure.org/databases/ (accessed Apr 25, 2017).

2. Snyder, B. E. R. et al. Chem. Rev. 2018, 118 (5), 2718-2768.

3. Knott, B. C. et al. ACS Catalysis. American Chemical Society February 2, 2018, pp 770-784.

4. Dědeček, J. et al. ChemSusChem. Wiley-VCH Verlag February 7, 2019, pp 556-576.

5. Vjunov, A. et al. J. Am. Chem. Soc. 2014, 136 (23), 82968306.

6. Zecchina, A. et al. Phys. Chem. Chem. Phys. 2007, 9 (27), 3483.

7. thyssenkrupp industrial solutions. EnviNOx ${ }^{\circledR}$ - reduce NOx emissions to a minium https://www.thyssenkruppindustrial-solutions.com/en/products-andservices/fertilizer-plants/nitrate-plants/envinox/ (accessed Aug 30, 2019).

8. Colombo, M. et al. In Catalysis Today; Elsevier, 2010; Vol 151, pp 223-230.

9. Martín, N. et al. ChemCatChem 2017, 9 (10), 1754-1757.

10. Long, R. Q. et al. Catal. Letters 2001, 74 (3-4), 201-205.
11. Sobalík, Z. et al. In Catalysis Today; Elsevier, 2002; Vol. 75, pp 347-351.

12. Chen, H. Y. et al. Phys. Chem. Chem. Phys. 2000, 2 (13), 3083-3090.

13. Janas, J. et al. Appl. Catal. B Environ. 2009, 91 (1-2), 113122.

14. Martín, N. et al. Chem. - A Eur. J. 2017, 23 (54), 134041341.

15. Frey, A. M. et al. Catal. Letters 2009, 130 (1-2), 1-8.

16. Krishna, K. et al. Catal. Today 2006, 114 (1), 23-30.

17. Liu, Z. et al. Microporous Mesoporous Mater. 2007, 104 (13), 159-170.

18. Høj, M. et al. Appl. Catal. B Environ. 2009, 93 (1-2), 166176.

19. Gao, F. et al. ACS Catal. 2016, 6 (5), 2939-2954.

20. Junhua, L. I. et al. Environ. Sci. Technol. 2010, 44 (5), 17991805.

21. Kucherov, A. V. et al. Catal. Letters 1998, 56 (4), 173-181.

22. Panov, G. I. et al. Appl. Catal. A, Gen. 1992, 82 (1), 31-36.

23. Ovanesyan, N. S. et al. Kinet. Catal. 1998, 39 (6), 792-797.

24. Hammond, C. et al. Angew. Chemie Int. Ed. 2012, 51 (21), 5129-5133.

25. Kessouri, A. et al. Res. Chem. Intermed. 2018, 44 (4), 24752487.

26. Li, Y. et al. Chem. 2017, pp 928-949.

27. Long, R. Q. et al. Chem. Commun. 2000, No. 17, 16511652

28. Russo, A. V et al. Water Sci. Technol. 2018, 77 (4), 939-947.

29. Stolyarova, I. V. et al. Russ. J. Appl. Chem. 2007, 80 (5), 746-753.

30. Huong Le, T. X. et al. Microporous Mesoporous Mater. 2019, 278, 64-69.

31. Snyder, B. E. R. et al. Nature 2016, 536 (7616), 317-321.

32. Suzuki, E. et al. Chem. Lett. 1988, 17 (6), 953-956.

33. Panov, G. I. et al. J. Mol. Catal. 1990, 61 (1), 85-97.

34. Sobolev, V. I. et al. J. Catal. 1993, 139 (2), 435-443.

35. Dubkov, K. A. et al. J. Catal. 2002, 207 (2), 341-352.

36. Starokon, E. V. et al. Top. Catal. 2003, 23 (1/4), 137-143.

37. Hensen, E. J. M. et al. J. Catal. 2003, 220 (2), 260-264.

38. Tabor, E. et al. Commun. Chem. 2019, 2 (1), 71.

39. Tabor, E. et al. Catal. Today 2011, 169 (1), 16-23.

40. Pirngruber, G. D. et al. Phys. Chem. Chem. Phys. 2006, 8 
(34), 3939-3950.

41. Pirngruber, G. D. et al. J. Phys. Chem. B 2006, 110 (37), 18104-18107.

42. Gao, F. et al. Appl. Catal. B Environ. 2015, 164, 407-419.

43. Benco, L. et al. J. Phys. Chem. C 2009, 113 (43), 1880718816 .

44. Hallaert, S. D. et al. Inorg. Chem. 2017, 56 (17), 1068110690.

45. Li, S. et al. ACS Catal. 2018, 8 (11), 10119-10130.

46. Snyder, B. E. R. et al. Proc. Natl. Acad. Sci. 2018, 115 (18), 4565-4570.

47. Rosa, A. et al. Inorg. Chem. 2010, 49 (8), 3866-3880.

48. Snyder, B. E. R. et al. Proc. Natl. Acad. Sci. 2018, 115 (48), 12124-12129.

49. Devos, J. et al. Chem. Mater. 2019, acs.chemmater.9b03738.

50. Bols, M. L. et al. J. Am. Chem. Soc. 2018, 140 (38), 1202112032.

51. Snyder, B. E. R. et al. J. Am. Chem. Soc. 2018, 140 (29), 9236-9243.

52. Vanelderen, P. et al. J. Am. Chem. Soc. 2015, 137 (19), 6383-6392.

53. Göltl, F. et al. ACS Catal. 2016, 6 (12), 8404-8409.

54. Pantu, P. et al. In Chemical Engineering Communications; Taylor \& Francis Group, 2008; Vol. 195, pp 1477-1485.

55. Szécsényi, Á. et al. ACS Catal. 2019, 9 (10), 9276-9284.

56. Notté, P. P. Top. Catal. 2000, 13 (4), 387-394.

57. Xia, H. et al. J. Phys. Chem. C 2008, 112 (24), 9001-9005.

58. Yamanaka, H. et al. J. Mol. Catal. A Chem. 2002, 178 (1-2), 89-95.

59. Han, L. et al. Chemical Reviews. American Chemical Society October 9, 2019, pp 10916-10976.

60 Brandenberger, S. et al. Catal. Rev. 2008, 50 (4), 492-531.

61. Doronkin, D. E. et al. Kinet. Catal. 2012, 53 (6), 747-752.

62. Starokon, E. V et al. J. Phys. Chem. C 2011, 115 (5), 21552161.

63. Zhao, G. et al. J. Phys. Chem. C 2019, 123 (45), 2743627447.

64. Zhao, G. et al. ACS Catal. 2020, 10 (2), 1406-1416.

65. Nobukawa, T. et al. J. Phys. Chem. B 2004, 108 (13), 40714079.

66. Wood, B. R. et al. J. Catal. 2004, 225 (2), 300-306.

67. Starokon, E. V et al. J. Catal. 2013, 300, 47-54.
68. Parfenov, M. V. et al. J. Catal. 2014, 318, 14-21.

69. Dubkov, K. A. et al. Kinet. Catal. 2001, 42 (2), 205-211.

70. Ivanov, D. P. et al. J. Catal. 2014, 311, 424-432.

71. Kiwi-Minsker, L. et al. J. Catal. 2003, 219 (2), 273-285.

72. Chen, P. et al. Reaction Chemistry and Engineering. 2019, pp 986-994.

73. Fahami, A. R. et al. React. Chem. Eng. 2019, 4 (6), 10001018.

74. Paolucci, C. et al. Science (80-. ). 2017, 357 (6354), 898903.

75. Lázár, K. et al. In Catalysis Today; Elsevier, 2005; Vol. 110, pp 239-246.

76. Gonzalez-Olmos, R. et al. Appl. Catal. B Environ. 2012, 125, 51-58.

77. Sashkina, K. A. et al. Microporous Mesoporous Mater. 2014.

78. Hammond, C. et al. ACS Catal. 2013, 3, 1835-1844.

79. Panov, G. I. et al. J. Catal. 2008, 254 (1), 110-120.

80. Que, L. et al. Acc. Chem. Res. 1996, 29 (4), 190-196.

81. Hammond, C. et al. ChemCatChem 2015, 7 (3), 434-440.

82. Lázár, K. et al. Hyperfine Interact. 2006, 167 (1-3), 779784.

83. Lázár, K. et al. Hyperfine Interact. 2008, 187 (1-3), 1-6.

84. Goldfarb, D. et al. J. Am. Chem. Soc. 1994, 116 (14), 63446353.

85. Patarin, J. et al. Zeolites 1992, 12 (1), 70-75.

86. Sun, K. et al. J. Phys. Chem. C 2008, 112 (41), 16036-16041.

87. Bordiga, S. et al. J. Catal. 1996, 158 (2), 486-501.

88. Gao, F. et al. ACS Catal. 2016, 6 (5), 2939-2954.

89. Di lorio, J. R. et al. Chem. Mater. 2016, 28 (7), 2236-2247.

90. Koebel, M. et al. Catal. Today 2000, 59 (3), 335-345.

91. Lambert, C. K. Reaction Chemistry and Engineering. The Royal Society of Chemistry May 28, 2019, pp 969-974.

92. Qi, G. et al. Catal. Letters 2008, 121 (1-2), 111-117.

93. Luo, J. Y. et al. Appl. Catal. B Environ. 2011, 102 (1-2), 110119.

94. Rivallan, M. et al. J. Catal. 2009, 264 (2), 104-116.

95. Janssens, T. V. W. et al. ACS Catal. 2015, 5 (5), 2832-2845. 